\title{
Influence of environmental factors on the life cycle and morphology of Artemia salina (Crustacea: Anostraca) in Sabkhet El Adhibet (SE Tunisia)
}

\author{
HACHEM BEN NACEUR, AMEL BEN REJEB JENHANI and MOHAMED SALAH ROMDHANE
}

Research Unit of Ecosystems and Aquatic Resources, National Institute of Agricultural Sciences of Tunisia, University of Carthage, 43 Av. Charles Nicolle 1082, Tunis Mahrajéne,Tunisia

Corresponding author: Hachem Ben Naceur, e-mail: hachem_b_naceur@yahoo.fr

(Received on 15 June 2008; Accepted on 7 May 2011)

\begin{abstract}
This study was aimed to examine in greater detail the influence of selected environmental factors on the life cycle and morphological characteristics of the brine shrimp Artemia salina (Linnaeus, 1758). During this follow-up, from November 2005 to April 2006 and from November 2006 to April 2007, Sabkhet El Adhibet (southeast Tunisia: $33^{\circ} 07^{\prime} 7.58^{\prime \prime} \mathrm{N}, 11^{\circ} 24^{\prime} 8.69^{\prime \prime} \mathrm{E}$ ) was surveyed monthly to determine the impact of water salinity, temperature, $\mathrm{pH}$, dissolved oxygen, and phytoplankton density and community structure on Artemia density, population structure, reproductive mode, and total offspring. Strong correlations were found between physicochemical parameters of water and Artemia reproduction characteristics. In contrast, no significant relationship was detected between physicochemical variables and Artemia population structure and density. Further, there were no correlations between phytoplankton density and the Artemia life cycle. Moreover, we observed relationships between physicochemical parameters and all morphological characteristics, especially between the width of $3^{\text {rd }}$ abdominal segment and salinity $\left(\mathrm{r}_{\mathrm{xy}}=0.96\right)$, temperature $\left(\mathrm{r}_{\mathrm{xy}}=0.73\right), \mathrm{pH}\left(\mathrm{r}_{\mathrm{xy}}=-0.77\right)$ and oxygen $\left(\mathrm{r}_{\mathrm{xy}}=-0.92\right)$ for male specimens, and between the length of the furca and both salinity $\left(\mathrm{r}_{\mathrm{xy}}=-0.76\right)$ and dissolved oxygen $\left(\mathrm{r}_{\mathrm{xy}}=0.74\right)$, and between the maximal diameter of compound eyes and temperature $\left(r_{x y}=-0.56\right)$ for female specimens. Principal component analysis (PCA) shows that male and female specimens collected at different environmental conditions converge, which explains the morphological similarity between them according to salinity, temperature, and dissolved oxygen concentration as well as total phytoplankton, diatom, cryptophyte, and dinophyte density.
\end{abstract}

Keywords: Artemia salina, physicochemical parameters, phytoplankton density, life cycle, morphological characteristics

\section{INTRODUCTION}

Permanent salt lakes show an almost continuous range of salinity, from brackish to hypersaline, and all except those at the highest salinities share some features, and perhaps stressors (e.g. oxygen and temperature), with their freshwater counterparts (JELLISON 2005). Inland hypersaline lakes in arid and semi-arid basins worldwide are 
relatively simple ecosystems, which can be used to understand how their components interact (GAJARDO et al. 2006). Studies on secondary production are essential for the evaluation of energy and matter transfer along the food web, as well as the rational management of aquatic ecosystems (DownING 1984). These challenging ecological settings make the genus Artemia useful model organisms for studies on evolutionary and ecological aspects of the stress response, at all levels of biological organization (Clegg \& Trotman 2002). Moreover, Artemia is considered to be an irreplaceable live food for the larval rearing of most marine fish and shellfish species (SorGELoos et al. 2001). Mariculture of finfish and crustaceans uses freshly-hatched nauplii of brine shrimp as part of the live food chain. In fact, the demand for Artemia cysts has gradually increased from a few metric tons to approximately 800 metric tons per annum, representing approximately $40 \%$ of the total aquaculture demand for earlystage feeds (Sorgeloos et al. 2001).

Organisms living in temporary inland and costal saline lakes (e.g. copepods, ostracods, rotifers, and branchiopods) have specific adaptive strategies for survival in high salinity conditions and for preventing the loss of cellular water under high osmolarity in hypersaline conditions. These aquatic invertebrates produce diapausing resistant stages in their life cycle, which allows them to survive during adverse periods or drought. While lower and intermediate salinity habitats are populated by various groups of invertebrates, hypersaline environments are characterised by monocultures of Artemia as major zooplankton (VAN STAPPEN 2002). In the natural environment, temperature, feeding conditions, and salinity are important factors influencing Artemia populations (Browne 1982; Wear \& Haslett 1987; CAmargo et al. 2004; LitvinENKo et al. 2007; ArASHKEVich et al. 2008). The impact of these ecological parameters can be explained through different Artemia responses, e.g. different reproductive strategies, life span, and morphological appearance.

Considering the significant role of Artemia in the food chain, as well as its importance in aquaculture, it was desirable to improve the understanding of the life cycle of the brine shrimp Artemia salina (Linnaeus, 1758). Hence, this research was aimed to identify the impact of some abiotic and biotic parameters on the density, reproductive mode, total offspring, and morphological parameters in the Artemia salina population from Sabkhet El Adhibet (SE Tunisia).

\section{MATERIAL AND METHODS}

\section{Study area}

Sabkha is a local Gulf Arabic word for a salt flat, and its geological usage implies intrasediment evaporate growth beneath a flat geomorphic surface with an elevation that is dictated by the top of the capillary fringe (WARREN \& Kendall 1985). The Tunisian territory contains a great number of sabkhas, especially in the centre and the south, representing $22 \%$ of the total wetland area (e.g. Sabkhet Sijoumi, Sabkhet El Kalbia, Sabkhet Sidi El Heni, Sabkhet El Melah of Zarzis, Sabkhet El Adhibet, and Sabkhet El Briga).

Sabkhet El Adhibet $\left(33^{\circ} 07^{\prime} 7.58^{\prime \prime} \mathrm{N}-11^{\circ} 24^{\prime} 8.69^{\prime \prime} \mathrm{E}\right)$ is an inland site, located in SE Tunisia, $16 \mathrm{~km}$ from the Tunisian-Libyan frontier. Its total surface is 12500 hectares, 
including 500 hectares occupied by industrial salt works. Trenches delimiting saltworks were dug artificially to consolidate the dividing dykes of the basins, with an average depth of $0.75 \mathrm{~m}$. These artificial canals in the borders of saltworks are filled by rainwater accumulated during the rainy season until May or June, and Artemia occurs there. In these canals, water salinity depends on precipitation and evaporation rate. The saltern is generally filled with rainwater from December to February. In Sabkhet El Adhibet, Artemia sp. was reported for the first time by RomDHANE et al. (2001). Lately, RoMDHANE et al. (2004) used discriminant analysis to compare the morphometry of Artemia males and females with other Artemia populations, and they concluded that Sabkhet El Adhibet is inhabit by Artemia salina (Linnaeus, 1758). MuÑoz et al. (2008) basing on the mitochondrial genetic diversity, confirmed this result.

Sabkhet El Adhibet is located in an upper-arid area. Maximum length and width are 8 and $7 \mathrm{~km}$, respectively. In the saltworks, water is pumped from the underground brine reservoir (salinity about $280 \mathrm{~g} \mathrm{~L}^{-1}$ ) and directly administered in the crystallizer. Three stations were selected (at the central parts of 3 sides of the saltwork, see Fig. 1) in artificial canals at the border of the saltworks (Artemia is absent in the crystallizers).

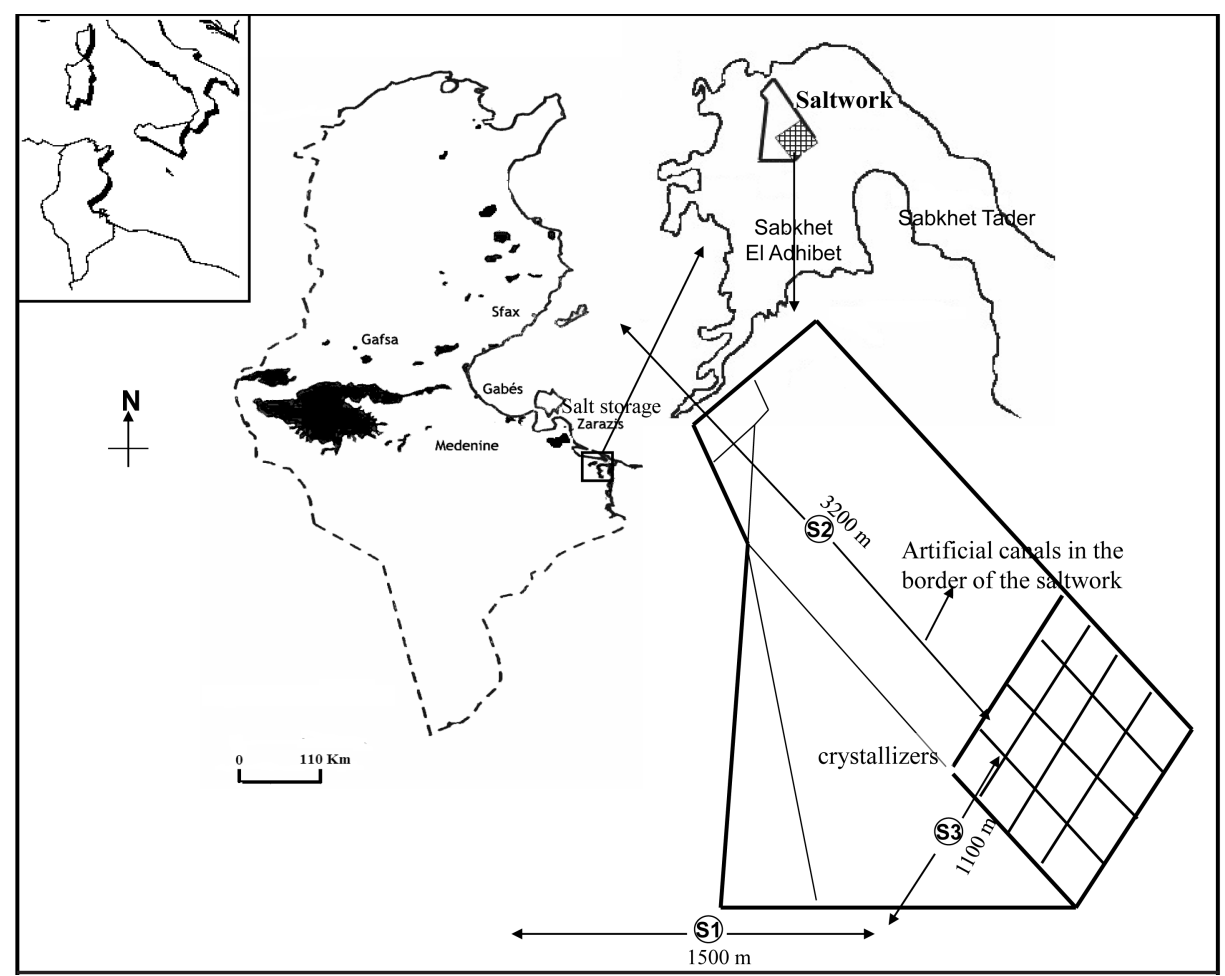

Fig. 1. Location of Sabkhet El Adhibet and sampling stations (S1-S3) 


\section{Sampling and measurements}

Variation in water temperature, salinity and $\mathrm{pH}$ was measured in situ by using a WTW handheld Multi-Parameter Instrument (Multi/340i/SET). These variables were monitored in the morning between 07:00 and 11:00 a.m., and sampling was performed monthly from November 2005 to April 2006 and from November 2006 to April 2007 (because the site was dry since May to late October). Dissolved oxygen concentration was determined by the Winkler test. During the investigation period, 1 L of water was sampled from the surface at each station and preserved with Lugol's solution and a neutralised formaldehyde solution for the determination of densities of microalgae. Their community structure was assessed in sedimentation chambers under an inverted microscope (Leitz).

Artemia samples were collected by filtering $100 \mathrm{~L}$ of water from each station through a plankton net (120 $\mu \mathrm{m}$ mesh size) and preserved in situ with neutralized 5\% formalin solution. Artemia density and population structure, fecundity, and type of offspring output (after dissecting ovigerous sacs) were assessed with a magnifying glass.

For the morphological analysis, male and female specimens were randomly collected by means of hand plankton nets (120 $\mu \mathrm{m}$ mesh size), only during the first study period (November 2005-April 2006) from station S3, in order to minimize differences in environmental influences (between the 3 stations) on morphological characteristics. Artemia biomass collected was stored in plastic containers and transferred to the laboratory for analysis. A random sample of 20 adult male and female specimens (i.e. well-developed antennae for males, and full and well-developed brood pouch, i.e. ovisac, for females) were removed and anesthetized with some droplets of water saturated with chloroform. The following morphological parameters were quantified in each Artemia sample: total length $(t l)$, abdomen length $(a l)$, width of $3^{\text {rd }}$ abdominal segment (wts), length of the furca $(l f)$, number of setae on the left furcal branch $(n l f)$, number of setae on the right furcal branch (nrf), width of the head (wh), maximal diameter of compound eyes $(d y)$, maximal distance between compound eyes $(d b y)$, length of $1^{\text {st }}$ antenna (la), width of the ovisac (wo) (for each female), width of $2^{\text {nd }}$ abdominal segment (wss) and width of the frontal knob $(f k)$ (for each male).

\section{Statistical methods}

The effect of the measured environmental parameters on the Artemia life cycle was analyzed using Pearson correlation coefficients calculated by XLSTAT-Pro 7.5 software.

Morphological characteristics were subjected to one-way ANOVA with post hoc Least Significant Difference (LSD) test using Statistica 5.0 software. Significance was accepted at $P<0.05$. Principal component analysis (PCA) was carried out using XLSTAT-Pro 7.5 software.

\section{RESULTS}

Results of physicochemical water parameters, phytoplankton characteristics, and the Artemia life cycle in Sabkhet El Adhibet used in this work were first pre- 
sented by BEN NACEUR et al. (2009). Table 1 reports the main information about biotic and abiotic conditions at this site.

\section{Impact on the Artemia life cycle}

Correlation analyses (Table 2) between environmental parameters (physicochemical parameters and phytoplankton density; $\left.\mathrm{X}_{1}-\mathrm{X}_{11}\right)$ and parameters of the $\mathrm{Ar}$ temia salina life cycle (Artemia population structure and density, reproductive mode, and offspring output; $\mathrm{Y}_{1}-\mathrm{Y}_{9}$ ) in Sabkhet El Adhibet revealed:

(1) a high negative correlation between:

- salinity $\left(\mathrm{X}_{1}\right)$ and offspring output $\left(\mathrm{Y}_{6}\right.$ and $\left.\mathrm{Y}_{7}\right)$ : number of cysts $\left(r_{\mathrm{xy}}=-0.75\right)$ and of nauplii per female $\left(r_{\mathrm{xy}}=-0.77\right)$;

- temperature $\left(\mathrm{X}_{2}\right)$ and offspring output $\left(\mathrm{Y}_{6}\right.$ and $\left.\mathrm{Y}_{7}\right)$ : number of cysts $\left(r_{\mathrm{xy}}=-0.69\right)$ and of nauplii per female $\left(r_{\mathrm{xy}}=-0.81\right)$;

$-\mathrm{pH}\left(\mathrm{X}_{3}\right)$ and oviparous reproduction mode $\left(\mathrm{Y}_{8} ; r_{\mathrm{xy}}=-0.71\right)$;

(2) a high positive correlation between:

$-\mathrm{pH}\left(\mathrm{X}_{3}\right)$ and ovoviviparous reproduction mode $\left(\mathrm{Y}_{9} ; r_{\mathrm{xy}}=0.71\right)$;

- cyanobacteria $\left(\mathrm{X}_{11}\right)$ and nauplii, juvenile and adult Artemia density $\left(\mathrm{Y}_{1}, \mathrm{Y}_{3}\right.$, and $\mathrm{Y}_{4} ; r_{\mathrm{xy}}=0.71,0.84$ and 0.90 , respectively);

(3) a moderate negative correlation between:

- salinity $\left(\mathrm{X}_{1}\right)$ and ovoviviparous reproduction mode $\left(\mathrm{Y}_{9} ; r_{\mathrm{xy}}=-0.67\right)$;

(4) a moderate positive correlation between:

- salinity $\left(\mathrm{X}_{1}\right)$ and oviparous reproduction mode $\left(\mathrm{Y}_{9} ; r_{\mathrm{xy}}=0.67\right)$;

$-\mathrm{pH}\left(\mathrm{X}_{3}\right)$ and offspring output $\left(\mathrm{Y}_{6}\right.$ and $\left.\mathrm{Y}_{7}\right)$ : number of cysts $\left(r_{\mathrm{xy}}=0.65\right)$ and nauplii per female $\left(r_{\mathrm{xy}}=0.70\right)$;

- dissolved oxygen $\left(\mathrm{X}_{4}\right)$ and offspring output $\left(\mathrm{Y}_{6}\right.$ and $\left.\mathrm{Y}_{7}\right)$ : number of cysts $\left(r_{\mathrm{xy}}=\right.$ $0.69)$ and of nauplii per female $\left(r_{\mathrm{xy}}=0.64\right)$.

\section{Impact on Artemia morphology}

The population sampled in April had significantly lower values of all morphometric characters (except for width of the ovisac in females), as compared to the other samples. The frontal knob of males and the diameter of compound eyes of both sexes, did not differ between samples (except for the April sample). For the other morphological characters, the statistical comparison (ANOVA, LSD test) shows different degrees of variation among samples, and did not show any particular similarity between Artemia specimens collected at different times (Table 3).

Table 4 shows the results of correlation analysis of the male morphological characteristics and physicochemical variables as well as phytoplankton density. The strongest correlation between morphological characteristics and physicochemical parameters was revealed between the width of $3^{\text {rd }}$ abdominal segment and salinity $\left(r_{\mathrm{xy}}=0.96\right)$, temperature $\left(r_{\mathrm{xy}}=0.73\right), \mathrm{pH}\left(r_{\mathrm{xy}}=-0.77\right)$, and oxygen $\left(r_{\mathrm{xy}}=-0.92\right)$. For phytoplankton density, the strongest correlation was between the length of the furca and density of total phytoplankton $\left(r_{\mathrm{xy}}=0.69\right)$, diatoms $\left(r_{\mathrm{xy}}=0.73\right)$, dinophytes $\left(r_{\mathrm{xy}}=\right.$ 0.73 ) and cryptophytes $\left(r_{\mathrm{xy}}=0.73\right.$ ). PCA shows according to axis 1 (with $53.06 \%$ of the total variance) that all specimens collected at different environmental conditions converge and show a morphological similarity in total length $(t l)$, maximal distance 


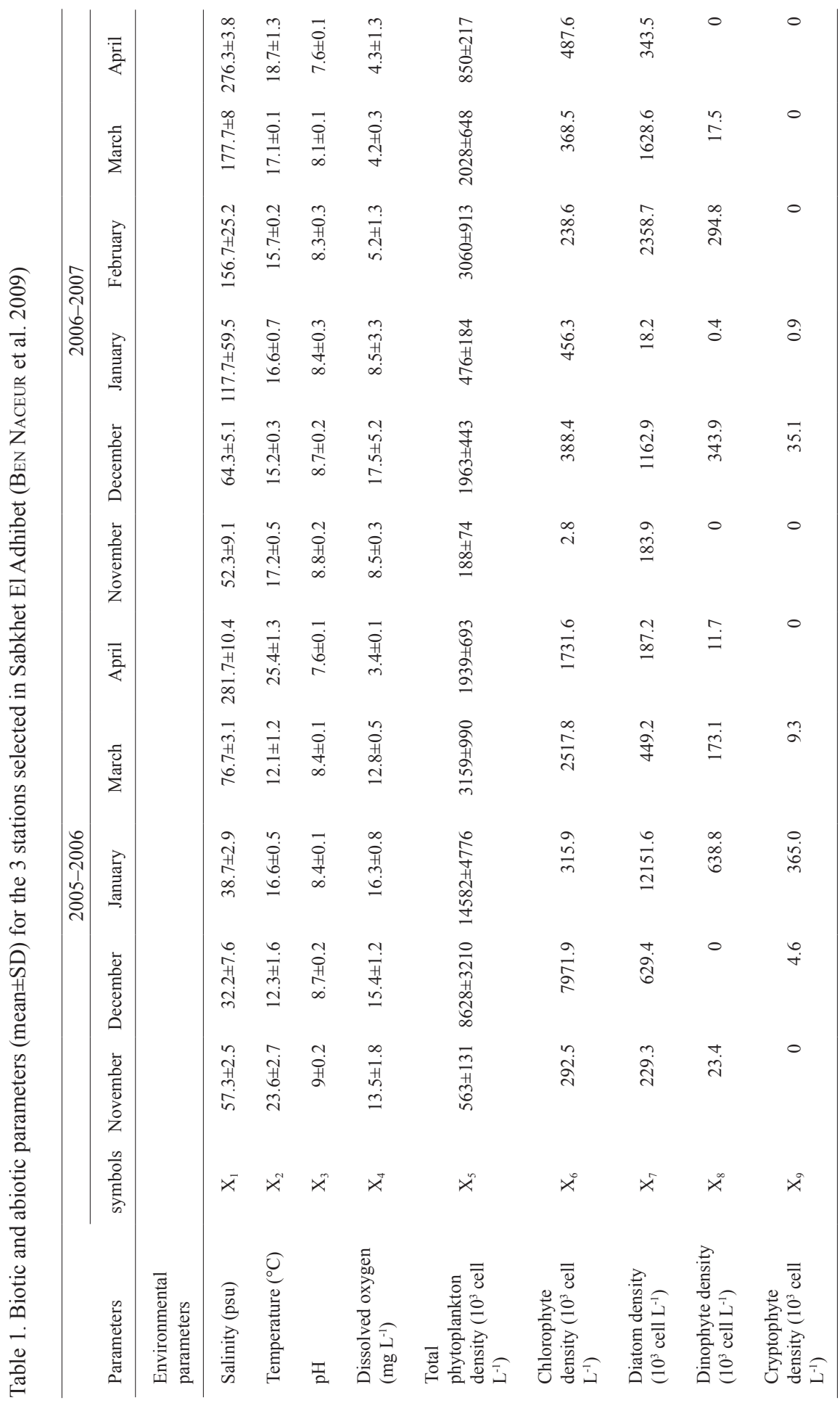




\begin{tabular}{|c|c|c|c|c|c|c|c|c|c|c|c|}
\hline 0 & $\stackrel{\circ}{\circ}$ & & 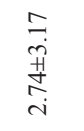 & $\begin{array}{l}\frac{O}{0} \\
\text { 贲 } \\
\text { - }\end{array}$ & $\begin{array}{l}\hat{\hat{~}} \\
\text { ํ. } \\
\text { ते } \\
\text { ô }\end{array}$ & 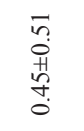 & 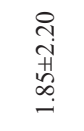 & 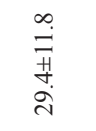 & 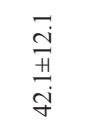 & $\Omega$ & $r$ \\
\hline 0 & $\stackrel{\circ}{\dot{ \pm}}$ & & 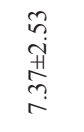 & 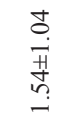 & $\begin{array}{l}\stackrel{0}{\hat{0}} \\
\text { 贲 } \\
\stackrel{n}{-}\end{array}$ & 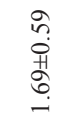 & 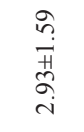 & 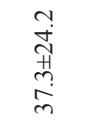 & $\begin{array}{l}m \\
\ddot{n} \\
\text { 1 } \\
\stackrel{0}{0} \\
\stackrel{n}{n}\end{array}$ & $\cong$ & $\hat{\sim}$ \\
\hline 0 & $\begin{array}{l}+ \\
\stackrel{0}{0} \\
\stackrel{0}{0}\end{array}$ & & $\begin{array}{l}\hat{o} \\
\dot{n} \\
\ddot{H} \\
\hat{n} \\
\dot{n}\end{array}$ & 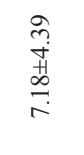 & 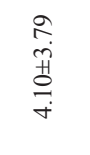 & 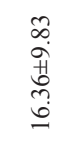 & $\begin{array}{l}\underset{J}{J} \\
\text { I } \\
\text { S } \\
=\end{array}$ & 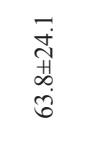 & 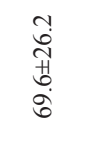 & $\cong$ & $\ddot{c}$ \\
\hline 0 & $\stackrel{\sigma}{\circ}$ & & 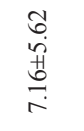 & $\begin{array}{l}\stackrel{7}{\exists} \\
\stackrel{H}{=} \\
=\end{array}$ & $\begin{array}{l}\text { तุ } \\
\stackrel{+}{\text { ते }} \\
\stackrel{-}{-}\end{array}$ & $\begin{array}{l}\vec{\sigma} \\
\dot{+} \\
+ \\
\ddot{b} \\
\dot{r}\end{array}$ & 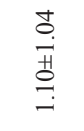 & $\begin{array}{l}\vec{c} \\
\text { ते } \\
\dot{q}\end{array}$ & 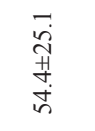 & 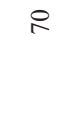 & 尺े \\
\hline 0 & ì & & $\begin{array}{l}m \\
\stackrel{-}{+} \\
\stackrel{+}{-} \\
\stackrel{0}{0}\end{array}$ & $\begin{array}{l}2 \\
0 \\
0 \\
\ddot{0} \\
\text { مे } \\
+\end{array}$ & 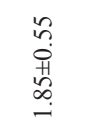 & \begin{tabular}{l}
$\infty$ \\
\multirow{0}{0}{} \\
$\stackrel{+}{0}$ \\
$\infty$ \\
0 \\
0
\end{tabular} & $\begin{array}{l}\text { ते } \\
0 \\
+1 \\
\text { مे } \\
\text { ì }\end{array}$ & 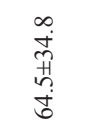 & 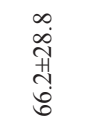 & $\stackrel{2}{2}$ & $\stackrel{\sim}{\sim}$ \\
\hline 0 & $\stackrel{+}{\leftrightarrows}$ & & $\begin{array}{l}\text { r } \\
\dot{0} \\
+ \\
+ \\
+ \\
r\end{array}$ & 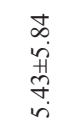 & $\begin{array}{l}\stackrel{n}{0} \\
0 \\
\stackrel{+}{ \pm} \\
0\end{array}$ & 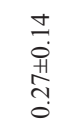 & $\begin{array}{l}\text { do } \\
\stackrel{+}{0} \\
\stackrel{H}{n} \\
-\end{array}$ & 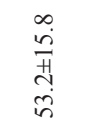 & 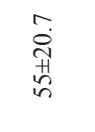 & $\approx$ & $\ddot{c}$ \\
\hline$\stackrel{\circ}{+}$ & $\stackrel{\circ}{+}$ & & $\begin{array}{l}\tilde{N} \\
\tilde{0} \\
0 \\
\infty \\
0 \\
0\end{array}$ & $\begin{array}{l}\infty \\
0 \\
0 \\
01 \\
0 \\
0 \\
0\end{array}$ & 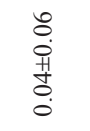 & $\begin{array}{l}n \\
0 \\
0 \\
⿱ \\
= \\
0\end{array}$ & $\begin{array}{l} \pm \\
0 \\
0 \\
+1 \\
\vdots \\
0\end{array}$ & $\frac{m}{\stackrel{m}{m}} \underset{m}{m}$ & $\begin{array}{l}m \\
\infty \\
\infty \\
\infty \\
\stackrel{+}{=}\end{array}$ & $\approx$ & in \\
\hline 0 & $\tilde{a}$ & & 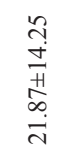 & $\begin{array}{l}n \\
\stackrel{0}{0} \\
01 \\
0 \\
0 \\
0\end{array}$ & 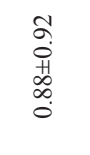 & 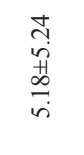 & 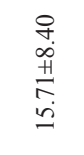 & $\begin{array}{l}0 \\
\stackrel{+}{+} \\
\mathbb{1} \\
\infty\end{array}$ & 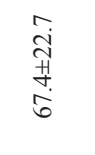 & $\infty$ & $\simeq$ \\
\hline $\begin{array}{l}\stackrel{+}{ \pm} \\
\stackrel{\Xi}{=}\end{array}$ & $\stackrel{\circ}{\therefore}$ & & $\begin{array}{l}\stackrel{o}{+} \\
\stackrel{\vec{H}}{0} \\
\stackrel{0}{0} \\
r\end{array}$ & 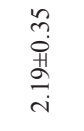 & $\begin{array}{l}= \\
0 \\
0 \\
0 \\
0 \\
0\end{array}$ & 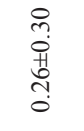 & $\begin{array}{l}\infty \\
\infty \\
\dot{+} \\
0 \\
\dot{0} \\
\dot{n}\end{array}$ & $\begin{array}{l}\text { ते } \\
\text { ते } \\
\text { iे } \\
\text { in }\end{array}$ & 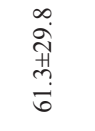 & $\therefore$ & $\tilde{\lambda}$ \\
\hline 0 & 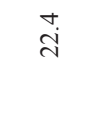 & & 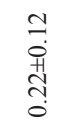 & $\begin{array}{l}\hat{0} \\
0 \\
01 \\
0 \\
0 \\
0\end{array}$ & $\begin{array}{l}1 \\
\stackrel{1}{0} \\
\text { D1 } \\
0 \\
0 \\
0\end{array}$ & 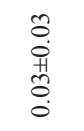 & $\begin{array}{l}5 \\
0 \\
0 \\
0 \\
0 \\
0\end{array}$ & 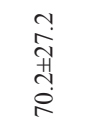 & 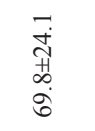 & $\cong$ & $\hat{\lambda}$ \\
\hline 0 & $\stackrel{\circ}{\infty}$ & & 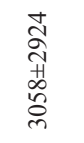 & 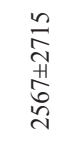 & 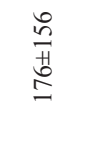 & $\begin{array}{l}\vec{J} \\
\vec{H} \\
\text { 品 }\end{array}$ & $\begin{array}{l}\vec{J} \\
\stackrel{H}{0} \\
\underline{-}\end{array}$ & $\begin{array}{l}\overrightarrow{+} \\
\vec{T} \\
\infty \\
+ \\
\dot{+}\end{array}$ & $\begin{array}{l}0 \\
\stackrel{+}{+} \\
\dddot{H} \\
\forall \\
i \\
i n\end{array}$ & $\stackrel{2}{\imath}$ & $\stackrel{\sim}{\sim}$ \\
\hline$\stackrel{0}{x}$ & $\overline{\bar{x}}$ & & $\bar{\nu}$ & $x^{2}$ & $\lambda^{m}$ & $\lambda^{+}$ & $\lambda^{n}$ & $\lambda^{0}$ & 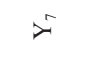 & $\lambda^{\infty}$ & $\lambda^{0}$ \\
\hline 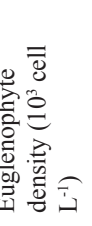 & 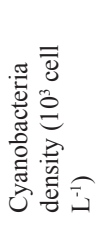 & 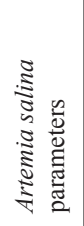 & 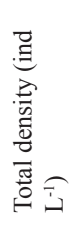 & 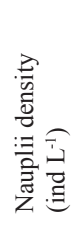 & 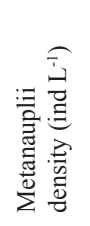 & 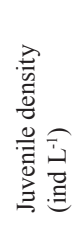 & 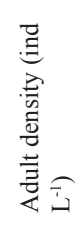 & 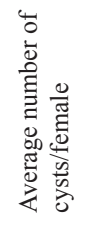 & 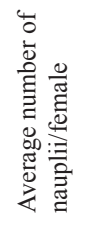 & 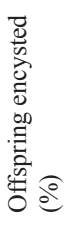 & 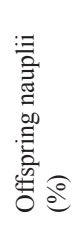 \\
\hline
\end{tabular}


Table 2. Pearson correlation matrix $\left(r_{\mathrm{xy}}\right)$ between environmental parameters and life cycle parameters (density, population structure, and reproduction) of Artemia salina. Bold numbers are significant at $P=0.05$. $\mathrm{X}_{1}$ : salinity; $\mathrm{X}_{2}$ : temperature; $\mathrm{X}_{3}: \mathrm{pH} ; \mathrm{X}_{4}$ : dissolved oxygen; $\mathrm{X}_{5}$ : total phytoplankton density; $\mathrm{X}_{6}$ : chlorophyte density; $\mathrm{X}_{7}$ : diatom density; $\mathrm{X}_{8}$ : dinophyte density; $\mathrm{X}_{9}$ : cryptophyte density; $\mathrm{X}_{10}$ : euglenophyte density; $\mathrm{X}_{11}$ : cyanobacteria density; $\mathrm{Y}_{1}$ : total Artemia density; $\mathrm{Y}_{2}$ : nauplii density; $\mathrm{Y}_{3}$ : metanauplii density; $\mathrm{Y}_{4}$ : juvenile density; $\mathrm{Y}_{5}$ : adult density; $\mathrm{Y}_{6}$ : average number of cysts/female; $\mathrm{Y}_{7}$ : average number of nauplii/female; $\mathrm{Y}_{8}$ : percent offspring encysted; $\mathrm{Y}_{9}$ : percent offspring nauplii

\begin{tabular}{llllllllllll}
\hline & $\mathrm{X}_{1}$ & $\mathrm{X}_{2}$ & $\mathrm{X}_{3}$ & $\mathrm{X}_{4}$ & $\mathrm{X}_{5}$ & $\mathrm{X}_{6}$ & $\mathrm{X}_{7}$ & $\mathrm{X}_{8}$ & $\mathrm{X}_{9}$ & $\mathrm{X}_{10}$ & $\mathrm{X}_{11}$ \\
\hline $\mathrm{Y}_{1}$ & -0.20 & -0.03 & 0.35 & 0.04 & -0.17 & -0.31 & -0.02 & 0.23 & -0.13 & -0.12 & $\mathbf{0 . 7 1}$ \\
$\mathrm{Y}_{2}$ & -0.31 & 0.45 & 0.58 & 0.22 & -0.25 & -0.29 & -0.10 & -0.10 & -0.10 & -0.10 & 0.16 \\
$\mathrm{Y}_{3}$ & 0.00 & -0.10 & 0.18 & -0.14 & -0.29 & -0.34 & -0.12 & 0.18 & -0.27 & -0.28 & $\mathbf{0 . 8 4}$ \\
$\mathrm{Y}_{4}$ & 0.08 & -0.24 & -0.01 & -0.27 & -0.12 & -0.18 & -0.03 & 0.21 & -0.19 & -0.17 & $\mathbf{0 . 9 0}$ \\
$\mathrm{Y}_{5}$ & -0.13 & -0.47 & 0.02 & 0.06 & 0.10 & -0.10 & 0.15 & 0.41 & 0.07 & 0.06 & 0.48 \\
$\mathrm{Y}_{6}$ & $\mathbf{- 0 . 7 5}$ & $\mathbf{- 0 . 6 9}$ & $\mathbf{0 . 6 5}$ & $\mathbf{0 . 6 9}$ & 0.44 & 0.44 & 0.21 & 0.47 & 0.18 & 0.13 & 0.39 \\
$\mathrm{Y}_{7}$ & $\mathbf{- 0 . 7 7}$ & $\mathbf{- 0 . 8 1}$ & $\mathbf{0 . 7 0}$ & $\mathbf{0 . 6 4}$ & 0.33 & 0.23 & 0.21 & 0.41 & 0.16 & 0.12 & 0.37 \\
$\mathrm{Y}_{8}$ & $\mathbf{0 . 6 7}$ & 0.39 & $\mathbf{- 0 . 7 1}$ & -0.36 & -0.14 & -0.02 & -0.15 & -0.12 & -0.09 & -0.08 & -0.17 \\
$\mathrm{Y}_{9}$ & $\mathbf{- 0 . 6 7}$ & -039 & $\mathbf{0 . 7 1}$ & 0.36 & 0.14 & 0.02 & 0.15 & 0.12 & 0.09 & 0.08 & 0.17 \\
\hline
\end{tabular}

between compound eyes $(d b y)$, diameter of compound eyes $(d y)$, width of $3^{\text {rd }}$ abdominal segment (wts), width of the head ( $w h)$ and width of $2^{\text {nd }}$ abdominal segment (wss) with a cumulative contribution of $62.7 \%$ (Table 5; Fig. 2).

For females (Table 4), the strongest correlations between the morphological characteristics and physicochemical parameters were revealed between the length of the furca and salinity $\left(r_{\mathrm{xy}}=-0.76\right)$, diameter of compound eyes and temperature $\left(r_{\mathrm{xy}}\right.$ $=-0.56)$, number of setae on left and right branches of the furca and $\mathrm{pH}\left(r_{\mathrm{xy}}=0.74\right.$ and 0.67 , respectively) and between dissolved oxygen and width of $3^{\text {rd }}$ abdominal segment $\left(r_{\mathrm{xy}}=0.7\right)$, length of the furca $\left(r_{\mathrm{xy}}=0.74\right)$, number of setae on the left furcal branch $\left(r_{\mathrm{xy}}=0.73\right)$, number of setae on the right furcal branch $\left(r_{\mathrm{xy}}=0.72\right)$, width of the head $\left(r_{\mathrm{xy}}=0.72\right)$, maximal diameter of compound eyes $\left(r_{\mathrm{xy}}=0.71\right)$ and length of $1^{\text {st }}$ antenna $\left(r_{\mathrm{xy}}=0.72\right)$. For densities of individual phytoplankton groups, the strongest correlation was between total length and densities of total phytoplankton $\left(r_{\mathrm{xy}}=\right.$ $0.62)$, diatoms $\left(r_{\mathrm{xy}}=0.65\right)$, dinophytes $\left(r_{\mathrm{xy}}=0.61\right)$, euglenophytes $\left(r_{\mathrm{xy}}=0.60\right)$, and cyanobacteria $\left(r_{\mathrm{xy}}=-0.40\right)$ and between abdomen length and densities of total phytoplankton $\left(r_{\mathrm{xy}}=0.70\right)$, diatoms $\left(r_{\mathrm{xy}}=0.70\right)$, dinophytes $\left(r_{\mathrm{xy}}=0.54\right)$, euglenophytes $\left(r_{\mathrm{xy}}\right.$ $=0.63)$, and cyanobacteria $\left(r_{\mathrm{xy}}=-0.52\right)$.

For males, PCA shows according to axis 1 (with $62.47 \%$ of the total variance) that all specimens collected at different environmental conditions converge and show morphological similarity in total length $(t)$, maximal distance between compound 
Table 3. Mean values (and SD in parentheses) of morphometric characters of male and female Artemia salina

Female

\begin{tabular}{|c|c|c|c|c|c|c|c|c|c|c|c|}
\hline & $t l$ & $a l$ & wo & $w t s$ & $l f$ & $n l f$ & $n r f$ & $w h$ & $d b y$ & $d y$ & la \\
\hline $\begin{array}{c}\text { November } \\
2005\end{array}$ & $\begin{array}{c}9.8^{\mathrm{b}} \\
(1.0)\end{array}$ & $\begin{array}{c}4.5^{\mathrm{b}} \\
(0.7)\end{array}$ & $\begin{array}{c}1.5^{\mathrm{a}} \\
(0.3)\end{array}$ & $\begin{array}{l}0.6^{\mathrm{bc}} \\
(0.1)\end{array}$ & $\begin{array}{c}0.3^{\mathrm{b}} \\
(0.0)\end{array}$ & $\begin{array}{c}6.9^{\mathrm{d}} \\
(1.7)\end{array}$ & $\begin{array}{c}6.4^{\mathrm{c}} \\
(1.9)\end{array}$ & $\begin{array}{c}0.9^{\mathrm{c}} \\
(0.1)\end{array}$ & $\begin{array}{c}1.4^{\mathrm{b}} \\
(0.2)\end{array}$ & $\begin{array}{l}0.3^{\mathrm{b}} \\
(0.0)\end{array}$ & $\begin{array}{l}0.7^{\mathrm{b}} \\
(0.1)\end{array}$ \\
\hline $\begin{array}{c}\text { December } \\
2005\end{array}$ & $\begin{array}{l}10.2^{b} \\
(1.2)\end{array}$ & $\begin{array}{c}5.6^{\mathrm{c}} \\
(0.6)\end{array}$ & $\begin{array}{c}1.9^{\mathrm{a}} \\
(0.4)\end{array}$ & $\begin{array}{c}0.6^{\mathrm{c}} \\
(0.1)\end{array}$ & $\begin{array}{c}0.3^{\mathrm{b}} \\
(0.1)\end{array}$ & $\begin{array}{c}5.8^{\mathrm{c}} \\
(1.7)\end{array}$ & $\begin{array}{c}6.0^{\mathrm{c}} \\
(1.7)\end{array}$ & $\begin{array}{c}0.9^{c} \\
(0.1)\end{array}$ & $\begin{array}{c}1.5^{\mathrm{b}} \\
(0.2)\end{array}$ & $\begin{array}{c}0.3^{\mathrm{b}} \\
(0.0)\end{array}$ & $\begin{array}{c}0.8^{\mathrm{b}} \\
(0.1)\end{array}$ \\
\hline $\begin{array}{c}\text { January } \\
2006\end{array}$ & $\begin{array}{l}12.1^{\mathrm{c}} \\
(0.9)\end{array}$ & $\begin{array}{c}6.5^{\mathrm{d}} \\
(0.7)\end{array}$ & $\begin{array}{c}2.3^{\mathrm{a}} \\
(0.2)\end{array}$ & $\begin{array}{c}0.7^{\mathrm{d}} \\
(0.1)\end{array}$ & $\begin{array}{c}0.4^{\mathrm{d}} \\
(0.1)\end{array}$ & $\begin{array}{c}5.6^{\mathrm{c}} \\
(1.7)\end{array}$ & $\begin{array}{l}5.8^{\mathrm{bc}} \\
(1.9)\end{array}$ & $\begin{array}{l}1.0^{\mathrm{d}} \\
(0.1)\end{array}$ & $\begin{array}{l}1.7^{\mathrm{c}} \\
(0.1)\end{array}$ & $\begin{array}{c}0.3^{\mathrm{b}} \\
(0.0)\end{array}$ & $\begin{array}{c}0.8^{\mathrm{c}} \\
(0.1)\end{array}$ \\
\hline $\begin{array}{c}\text { March } \\
2006\end{array}$ & $\begin{array}{c}9.8^{\mathrm{b}} \\
(1.8)\end{array}$ & $\begin{array}{l}4.2^{\mathrm{ab}} \\
(1.2)\end{array}$ & $\begin{array}{c}1.8^{\mathrm{a}} \\
(0.5)\end{array}$ & $\begin{array}{c}0.5^{\mathrm{b}} \\
(0.1)\end{array}$ & $\begin{array}{c}0.3^{\mathrm{c}} \\
(0.1)\end{array}$ & $\begin{array}{c}4.5^{\mathrm{b}} \\
(1.8)\end{array}$ & $\begin{array}{l}4.8^{b} \\
(2.1)\end{array}$ & $\begin{array}{l}0.7^{\mathrm{b}} \\
(0.1)\end{array}$ & $\begin{array}{c}1.5^{\mathrm{b}} \\
(0.3)\end{array}$ & $\begin{array}{l}0.3^{\mathrm{b}} \\
(0.1)\end{array}$ & $\begin{array}{c}0.7^{\mathrm{b}} \\
(0.2)\end{array}$ \\
\hline April 2006 & $\begin{array}{c}7.7^{\mathrm{a}} \\
(0.5)\end{array}$ & $\begin{array}{c}3.9^{\mathrm{a}} \\
(0.4)\end{array}$ & $\begin{array}{c}1.9^{\mathrm{a}} \\
(2.7)\end{array}$ & $\begin{array}{c}0.4^{\mathrm{a}} \\
(0.1)\end{array}$ & $\begin{array}{c}0.1^{\mathrm{a}} \\
(0.0)\end{array}$ & $\begin{array}{c}1.5^{\mathrm{a}} \\
(0.8)\end{array}$ & $\begin{array}{c}1.4^{\mathrm{a}} \\
(0.7)\end{array}$ & $\begin{array}{c}0.6^{\mathrm{a}} \\
(0.0)\end{array}$ & $\begin{array}{c}1.0^{\mathrm{a}} \\
(0.1)\end{array}$ & $\begin{array}{c}0.2^{\mathrm{a}} \\
(0.0)\end{array}$ & $\begin{array}{c}0.5^{\mathrm{a}} \\
(0.1)\end{array}$ \\
\hline
\end{tabular}

Male

\begin{tabular}{|c|c|c|c|c|c|c|c|c|c|c|c|c|}
\hline & $t l$ & $a l$ & wss & $w t s$ & lf & $n l f$ & $n r f$ & $w h$ & $d b y$ & $d y$ & la & $f k$ \\
\hline $\begin{array}{l}\text { November } \\
2005\end{array}$ & $\begin{array}{c}8.4^{\mathrm{b}} \\
(0.7)\end{array}$ & $\begin{array}{l}4.1^{\mathrm{ab}} \\
(0.4)\end{array}$ & $\begin{array}{c}0.8^{\mathrm{e}} \\
(0.1)\end{array}$ & $\begin{array}{c}0.6^{\mathrm{c}} \\
(0.1)\end{array}$ & $\begin{array}{c}0.4^{\mathrm{b}} \\
(0.1)\end{array}$ & $\begin{array}{l}10.0^{\mathrm{d}} \\
(3.1)\end{array}$ & $\begin{array}{c}9.7^{\mathrm{c}} \\
(3.1)\end{array}$ & $\begin{array}{c}0.8^{\mathrm{c}} \\
(0.1)\end{array}$ & $\begin{array}{c}1.6^{\mathrm{b}} \\
(0.2)\end{array}$ & $\begin{array}{c}0.4^{\mathrm{b}} \\
(0.0)\end{array}$ & $\begin{array}{c}1.0^{\mathrm{b}} \\
(0.2)\end{array}$ & $\begin{array}{l}0.2^{\mathrm{b}} \\
(0.0)\end{array}$ \\
\hline $\begin{array}{l}\text { December } \\
2005\end{array}$ & $\begin{array}{c}8.9^{\mathrm{b}} \\
(0.9)\end{array}$ & $\begin{array}{l}4.5^{\mathrm{bc}} \\
(0.5)\end{array}$ & $\begin{array}{c}0.6^{\mathrm{b}} \\
(0.1)\end{array}$ & $\begin{array}{c}0.5^{\mathrm{b}} \\
(0.1)\end{array}$ & $\begin{array}{c}0.3^{\mathrm{b}} \\
(0.1)\end{array}$ & $\begin{array}{l}8.1^{\mathrm{c}} \\
(2.3)\end{array}$ & $\begin{array}{c}7.6^{\mathrm{b}} \\
(2.4)\end{array}$ & $\begin{array}{c}0.8^{\mathrm{c}} \\
(0.1)\end{array}$ & $\begin{array}{c}1.7^{\mathrm{b}} \\
(0.2)\end{array}$ & $\begin{array}{c}0.4^{\mathrm{b}} \\
(0.0)\end{array}$ & $\begin{array}{l}1.1^{\mathrm{c}} \\
(0.1)\end{array}$ & $\begin{array}{c}0.2^{\mathrm{b}} \\
(0.0)\end{array}$ \\
\hline $\begin{array}{c}\text { January } \\
2006\end{array}$ & $\begin{array}{c}9.7^{\mathrm{c}} \\
(1.4)\end{array}$ & $\begin{array}{c}4.7^{\mathrm{c}} \\
(0.8)\end{array}$ & $\begin{array}{c}0.7^{\mathrm{c}} \\
(0.1)\end{array}$ & $\begin{array}{c}0.5^{\mathrm{b}} \\
(0.1)\end{array}$ & $\begin{array}{c}0.8^{\mathrm{c}} \\
(0.3)\end{array}$ & $\begin{array}{l}5.9^{\mathrm{ab}} \\
(2.8)\end{array}$ & $\begin{array}{c}6.7^{\mathrm{b}} \\
(3.0)\end{array}$ & $\begin{array}{c}0.8^{\mathrm{c}} \\
(0.1)\end{array}$ & $\begin{array}{c}1.6^{\mathrm{b}} \\
(0.2)\end{array}$ & $\begin{array}{c}0.4^{\mathrm{b}} \\
(0.1)\end{array}$ & $\begin{array}{l}1.1^{\mathrm{c}} \\
(0.2)\end{array}$ & $\begin{array}{c}0.2^{\mathrm{b}} \\
(0.0)\end{array}$ \\
\hline $\begin{array}{c}\text { March } \\
2006\end{array}$ & $\begin{array}{l}9.1^{\mathrm{bc}} \\
(1.8)\end{array}$ & $\begin{array}{l}4.4^{\mathrm{bc}} \\
(1.0)\end{array}$ & $\begin{array}{c}0.7^{\mathrm{c}} \\
(0.2)\end{array}$ & $\begin{array}{c}0.5^{\mathrm{b}} \\
(0.1)\end{array}$ & $\begin{array}{c}0.4^{\mathrm{b}} \\
(0.1)\end{array}$ & $\begin{array}{c}6.3^{\mathrm{b}} \\
(2.4)\end{array}$ & $\begin{array}{l}6.2^{\mathrm{ab}} \\
(2.4)\end{array}$ & $\begin{array}{c}0.7^{\mathrm{b}} \\
(0.1)\end{array}$ & $\begin{array}{l}1.7^{\mathrm{b}} \\
(0.4)\end{array}$ & $\begin{array}{c}0.4^{\mathrm{b}} \\
(0.1)\end{array}$ & $\begin{array}{l}1.1^{\mathrm{c}} \\
(0.1)\end{array}$ & $\begin{array}{l}0.2^{\mathrm{b}} \\
(0.0)\end{array}$ \\
\hline April 2006 & $\begin{array}{c}7.5^{\mathrm{a}} \\
(0.6)\end{array}$ & $\begin{array}{c}3.8^{\mathrm{a}} \\
(0.3)\end{array}$ & $\begin{array}{c}0.4^{\mathrm{a}} \\
(0.0)\end{array}$ & $\begin{array}{c}0.3^{\mathrm{a}} \\
(0.2)\end{array}$ & $\begin{array}{c}0.2^{\mathrm{a}} \\
(0.0)\end{array}$ & $\begin{array}{c}4.6^{\mathrm{a}} \\
(2.3)\end{array}$ & $\begin{array}{c}4.6^{\mathrm{a}} \\
(2.3)\end{array}$ & $\begin{array}{c}0.6^{\mathrm{a}} \\
(0.1)\end{array}$ & $\begin{array}{c}1.2^{\mathrm{a}} \\
(0.1)\end{array}$ & $\begin{array}{c}0.3^{\mathrm{a}} \\
(0.0)\end{array}$ & $\begin{array}{c}0.8^{\mathrm{a}} \\
(0.2)\end{array}$ & $\begin{array}{c}0.2^{\mathrm{a}} \\
(0.0)\end{array}$ \\
\hline
\end{tabular}

Same letters show non-significant differences within rows $(P=0.05)$. Characters: $t l=$ total length; $a l$ $=$ abdomen length; $w s s=$ width of 2 nd abdominal segment; $w t s=$ width of $3 \mathrm{rd}$ abdominal segment; $l f=$ length of furca; $n l f=$ number of setae on left furcal branch; $n r f=$ number of setae on right furcal branch; $w h=$ width of head; $d y=$ diameter of compound eyes; $d b y=$ maximal distance between compound eyes; $l a=$ length of 1 st antenna; $f k=$ width of the frontal knob; $w o=$ width of ovisac. 
Table 4. Pearson correlation matrix $\left(r_{\mathrm{xy}}\right)$ between environmental parameters and adult morphological characteristics of Artemia salina. Bold numbers are significant at $P=0.05$

\begin{tabular}{|c|c|c|c|c|c|c|c|c|c|c|c|}
\hline \multicolumn{12}{|c|}{ Males } \\
\hline & $X_{1}$ & $\mathrm{X}_{2}$ & $\mathrm{X}_{3}$ & $\mathrm{X}_{4}$ & $\mathrm{X}_{5}$ & $\mathrm{X}_{6}$ & $\mathrm{X}_{7}$ & $\mathrm{X}_{8}$ & $\mathrm{X}_{9}$ & $\mathrm{X}_{10}$ & $\mathrm{X}_{11}$ \\
\hline$t l$ & -0.47 & -0.43 & 0.24 & 0.45 & 0.37 & -0.16 & 0.39 & 0.41 & 0.35 & 0.18 & -0.29 \\
\hline al & -0.35 & -0.35 & 0.15 & 0.34 & 0.35 & -0.02 & 0.35 & 0.33 & 0.31 & 0.10 & -0.29 \\
\hline wss & -0.61 & -0.60 & 0.33 & 0.58 & 0.40 & -0.26 & 0.43 & 0.51 & 0.38 & 0.37 & -0.33 \\
\hline wts & 0.96 & 0.73 & -0.77 & -0.92 & -0.29 & 0.27 & -0.35 & -0.35 & -0.25 & -0.43 & 0.07 \\
\hline If & -0.55 & -0.31 & 0.27 & 0.59 & 0.69 & -0.39 & 0.73 & 0.73 & 0.73 & -0.12 & -0.47 \\
\hline$n l f$ & -0.38 & -0.02 & 0.56 & 0.39 & -0.18 & -0.13 & -0.13 & -0.21 & -0.17 & -0.02 & 0.41 \\
\hline$n r f$ & -0.39 & -0.01 & 0.52 & 0.41 & -0.06 & -0.20 & -0.01 & -0.08 & -0.04 & -0.07 & 0.30 \\
\hline$w h$ & -0.68 & -0.32 & 0.64 & 0.69 & 0.21 & -0.23 & 0.27 & 0.19 & 0.21 & 0.06 & 0.06 \\
\hline$d y$ & -0.55 & -0.44 & 0.49 & 0.52 & 0.06 & -0.10 & 0.09 & 0.08 & 0.02 & 0.32 & 0.05 \\
\hline$d b y$ & -0.55 & -0.38 & 0.47 & 0.54 & 0.17 & -0.15 & 0.21 & 0.18 & 0.15 & 0.19 & -0.01 \\
\hline la & -0.48 & -0.49 & 0.27 & 0.46 & 0.31 & -0.07 & 0.32 & 0.33 & 0.27 & 0.27 & -0.25 \\
\hline$f k$ & -0.65 & -0.47 & 0.51 & 0.64 & 0.27 & -0.21 & 0.31 & 0.30 & 0.25 & 0.23 & -0.10 \\
\hline \multicolumn{12}{|c|}{ Females } \\
\hline$t l$ & -0.66 & -0.45 & 0.38 & 0.68 & 0.62 & -0.23 & 0.65 & 0.61 & 0.60 & 0.00 & $-\mathbf{- 0 . 4 0}$ \\
\hline al & -0.50 & -0.39 & 0.20 & 0.54 & 0.70 & 0.07 & 0.70 & 0.54 & 0.63 & -0.16 & -0.52 \\
\hline Wo & -0.01 & -0.06 & -0.08 & 0.02 & 0.19 & 0.02 & 0.18 & 0.17 & 0.18 & -0.04 & -0.19 \\
\hline$w t s$ & -0.68 & -0.41 & 0.46 & 0.70 & 0.54 & -0.23 & 0.58 & 0.51 & 0.53 & -0.02 & -0.29 \\
\hline lf & -0.76 & -0.55 & 0.51 & 0.74 & 0.46 & -0.40 & 0.52 & 0.56 & 0.48 & 0.24 & -0.27 \\
\hline nlf & -0.72 & -0.28 & 0.74 & 0.73 & 0.15 & -0.27 & 0.21 & 0.13 & 0.15 & 0.02 & 0.17 \\
\hline$n r f$ & -0.71 & -0.37 & 0.67 & 0.72 & 0.19 & -0.22 & 0.25 & 0.18 & 0.18 & 0.10 & 0.08 \\
\hline$w h$ & -0.68 & -0.34 & 0.50 & 0.72 & 0.56 & -0.20 & 0.60 & 0.48 & 0.54 & -0.14 & -0.26 \\
\hline$d y$ & -0.72 & -0.56 & 0.47 & 0.71 & 0.47 & -0.23 & 0.51 & 0.50 & 0.45 & 0.21 & -0.30 \\
\hline$d b y$ & -0.58 & -0.41 & 0.45 & 0.56 & 0.23 & -0.23 & 0.27 & 0.28 & 0.22 & 0.22 & -0.08 \\
\hline la & -0.72 & -0.49 & 0.54 & 0.72 & 0.40 & -0.25 & 0.44 & 0.41 & 0.38 & 0.16 & -0.18 \\
\hline
\end{tabular}

For abbreviations, see Tables 1 and 3. 

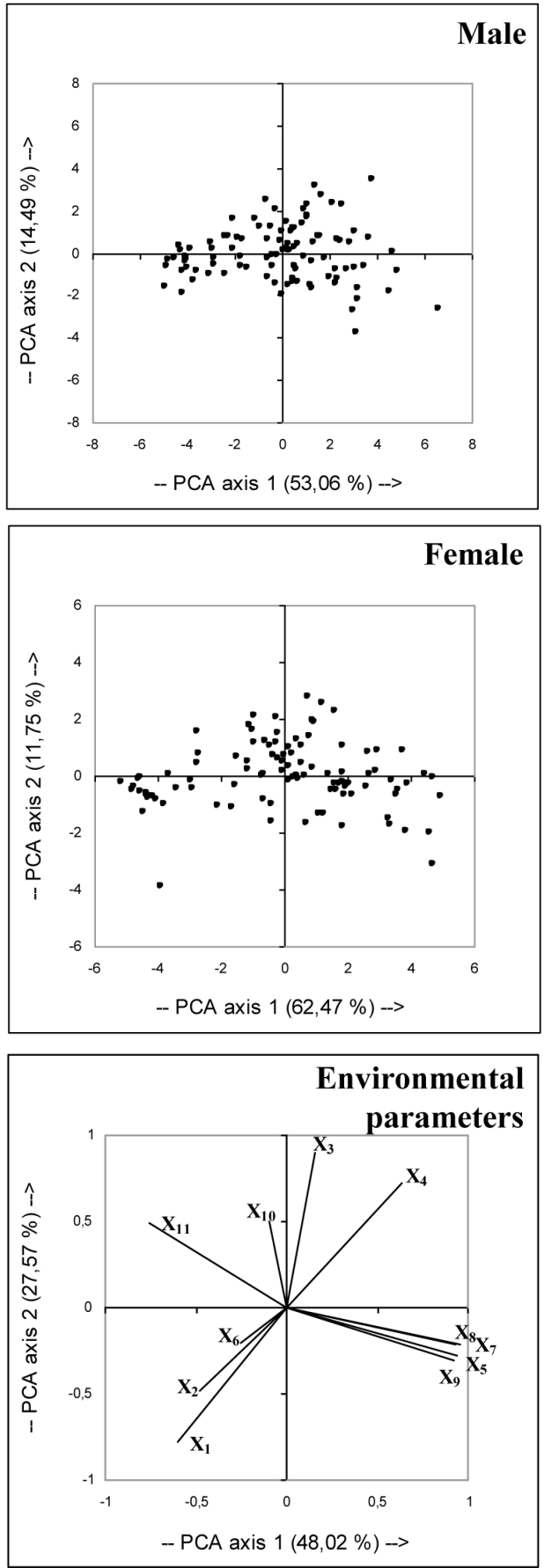

Fig. 2. Principal component analysis (PCA) of morphological characteristics of adult Artemia salina and environmental parameters studied. For abbreviations, see Table 1 
Table 5. Contributions of principal components F1 and F2 of physicochemical parameters to the variance of adult morphological characters of Artemia salina

\begin{tabular}{lrrrr}
\hline \multirow{2}{*}{ Morphological character } & \multicolumn{2}{c}{ Males } & \multicolumn{2}{c}{ Females } \\
\cline { 2 - 5 } & 10.886 & 9.447 & 12.487 & 4.121 \\
\hline Total length & 8.344 & 12.277 & 8.317 & 7.286 \\
Abdomen length & 9.110 & 0.915 & - & - \\
Width of 2nd abdominal segment & - & - & 0.463 & 15.934 \\
Width of ovisac & 9.546 & 1.926 & 11.624 & 0.302 \\
Width of 3rd abdominal segment & 5.723 & 4.470 & 10.474 & 0.001 \\
Length of furca & 4.632 & 31.812 & 6.322 & 34.495 \\
No. of setae on left furcal branch & 4.343 & 34.695 & 6.260 & 33.795 \\
No. of setae on right furcal branch & 9.563 & 1.157 & 10.769 & 0.075 \\
Width of head & 12.172 & 0.072 & 12.698 & 0.788 \\
Maximal distance between & 11.423 & 0.602 & 9.302 & 3.191 \\
compound eyes & 5.319 & 1.411 & 11.285 & 0.012 \\
Diameter of compound eyes & 8.937 & 1.219 & & - \\
Length of 1st antenna & & & & - \\
Width of frontal knob & & & &
\end{tabular}

eyes ( $d b y)$, width of 3rd abdominal segment (wts), length of 1st antenna (la), width of the head $(w h)$, and length of the furca $(l f)$, with a cumulative contribution of $69.33 \%$ (Table 5; Fig. 2).

Considering the impact of biotic and abiotic parameters on the Artemia morphological structure, it is clear that salinity, temperature, and dissolved oxygen for physicochemical parameters of water, as well as densities of total phytoplankton, diatoms, cryptophytes, and dinophytes, were the major factors affecting Artemia morphology (Fig. 2).

\section{DISCUSSION}

Sabkhet El Adhibet is an ephemeral site, depending totally on rainfall. Generally, high salinity is the norm in this ecosystem; low salinities coincide with rainfall, as freshwater runs over the flats. In summer, this semi-arid ecosystem is hot and dry, with salt crystals covering the sediment surface. Moreover, the flats are heterogeneous, exhibiting spatial and temporal variability in salinity, temperature, $\mathrm{pH}$, dissolved oxygen, and microalgae density affecting zooplankton biodiversity. GLIWICz et al. (1995) 
reported that, in spite of the simplicity of the trophic structure (short food chain) and the limited biodiversity of this ecosystem, it is difficult to assess the consequences of abiotic fluctuations and shifts in phytoplankton concentration/composition on the Artemia franciscana population.

In natural environments, temperature, feeding conditions, and salinity are important factors influencing Artemia populations (WEAR \& HasletT 1987; VAN StaPPEN et al. 2001; TORRENTERA \& Dodson 2004). Our study of the correlations between environmental parameters and Artemia populations in Sabkhet El Adhibet revealed that physicochemical parameters present significant connections with the reproductive mode and offspring output. These results confirm those reported by CAMARGO et al. (2004) and Torrentera \& Dodson (2004). In fact, induction of diapause in Artemia may be under maternal control in response to environmental conditions that determine the metabolic state of the mature embryo (MARCus 1984; GAJARDO \& BEARDMORE 1989). BAid (1967), VANHEACKe et al. (1984), Triantaphyllidis et al. (1995) and TORRENTERA \& DODSON (2004) reported that the termination of diapause in Artemia and the selection of reproductive mode are mainly under environmental control. CAMARGO et al. (2004) state that the variation in physicochemical conditions of some thalassohaline (marine) sites in the Colombian Caribbean did not influence Artemia biomass production. Conversely, TORRENTERA \& DODSON (2004), who studied the ecology of the brine shrimp Artemia in the Yucatan (Mexico) salterns, showed that the Artemia population dynamics and abundance are highly influenced by environmental factors, principally oxygen, salinity, and temperature. Furthermore, CAMARGO et al. (2004) reported that the reproductive experiment (mean cyst production per female) does not entirely agree with the estimated cyst production potential but may be due to a combination of certain parameters (i.e. salinity, oxygen concentration, low nitrate, and starvation of the adult Artemia population after reaching a high density). In our case, the analysis of physicochemical parameters and Artemia densities in Sabkhet El Adhibet did not reveal any correlation between them, confirming the findings of CAMARGO et al. (2004).

Phytoplankton studies and monitoring are useful for the control of the physicochemical and biological conditions of the water. The dynamics of phytoplankton is influenced many of the environmental processes that affect species diversity. D'Agostino \& Provasoli (1968) recognized that food quality and quantity could induce in Artemia the oviparity reproduction mode. NEwMAn (2001, cited in CAMARGo et al. 2004) reported that food density might be the determining factor for Artemia to select the oviparous mode of reproduction rather than food quality. Moreover, CAMARGO et al. (2004) found that chlorophyll $a$ was negatively correlated to cyst production, potentially supporting the hypothesis reported by BALLARDIN \& METALLI (1963), D’Agostino \& Provasoli (1968), Amat (1985), and Román \& Rodríguez (1986), that insufficient food plays an important role in cyst production. On the other hand, LENZ (1987) observed that zooplankton population dynamics are influenced by abiotic factors (salinity, temperature, and nutrient concentration) and by biological interactions (predation, competition, and grazers). Sorgeloos et al. (1986) reported that the best conditions for Artemia biomass production are at the lower salinity levels (100 ppt) and under conditions of very regular food availability. 
DOLAPSAKIS et al. (2005) showed that the density of the microalga Dunaliella salina (Chlorophyta) reached its minimum in summer, when grazing by Artemia parthenogenetica and Fabrea salina was intense. During our survey, the statistical analyses did not show any correlation between phytoplankton density and the Artemia life cycle (density, population structure, and reproduction). The absence of connections between Artemia and phytoplankton density could be explained by the fact that in Sabkhet El Adhibet and during a certain period (especially when salinity did not exceed $70 \mathrm{~g} \mathrm{~L}^{-1}$ ), Artemia coexisted with some other zooplankton (e.g. Branchiopoda, Cladocera, Rotifera) but studied herein as a monoculture, and then the impact of the other species on phytoplankton density was not considered.

Hypersaline habitats are abundant worldwide and provide important models for studying adaptation of aquatic organisms to extreme environments. Biotopes inhabited by Artemia are often characterized by extreme ecological conditions. The formation of body proportions in the brine shrimp is affected by many abiotic factors, such as temperature, ion ratio, oxygen content, acidity, and general mineralization (LitVinenKo \& Boyko 2008). According to previous studies (e.g. GaevsKaya 1916; GiLCHRIST 1960; AMAT 1980; LitVINENKO et al. 2007), salinity and salt composition are the most important ecological characteristics affecting morphological and biometrical Artemia parameters. However, there are few published data about the impact of the other water physicochemical parameters on Artemia morphology.

In the present study, significant differences among adult specimen samples have been demonstrated for several morphological parameters. Specimens harvested in April (corresponding to the highest salinity) show the smallest sizes for the different morphological characteristics. Correlation analysis between different Artemia morphological characteristics and the different environmental parameters revealed that physicochemical water characteristics were correlated with all morphological parameters except width of the ovisac. For phytoplankton density, the results show a positive connection between total phytoplankton density and some morphological characteristics. Our data indicate that salinity and temperature were negatively connected with morphological characteristics, whereas $\mathrm{pH}$, oxygen concentration, and total phytoplankton density were positively connected with these parameters. The impact of salinity on Artemia morphology was observed by several researchers (Amat 1982; Amat et al. 1991; Naegel \& Rodriguez 2002). Naegel \& Rodriguez (2002) mentioned that the main reason for the decrease in size of adults is because at high salinity levels (200-250 $\left.\mathrm{g} \mathrm{L}^{-1}\right)$, food becomes a limiting factor and Artemia needs more energy for osmoregulation. BERTALANFFY \& KRYWIENCZYK (1953), studying the oxygen consumption of Artemia at one salinity level, revealed that respiration is proportional to the square of body length, and thus to surface area.

PCA revealed that salinity, temperature and oxygen are the physicochemical parameters that affect the largest number of morphological characteristics. In fact, Fig. 2 shows that for samples harvested at lower salinity, adult Artemia differ more strongly in their morphological characteristics than those harvested at high salinity (in April at salinity level $285 \mathrm{~g} \mathrm{~L}^{-1}$ ). These differences were expressed by a dispersed distribution of specimens collected at lower salinity, whereas the distribution of specimens collected at high salinity is more clumped. The ability of Artemia to change its 
appearance under the influence of salinity has been established by several authors, GaEvskaya (1916), rearing Artemia at different salinities, concluded that increasing salinity results in a reduction of adult brine shrimp body size and of the last abdominal segment (furca). Амат (1980) observed that Artemia living in natural environments is usually smaller than when cultured in the laboratory. Further, LiTVINENKo et al. (2007) revealed that only the number of setae on each furcal branch was changed as a function of salinity. In our morphological follow-up, the correlation analysis showed that salinity has an impact on all morphological structures.

In conclusion, this study allowed us to show relationships between water physicochemical parameters and Artemia reproduction characteristics. In contrast, no significant relationship was found between any physicochemical variable and Artemia population structure and density. Further, there was no correlation between phytoplankton density and the Artemia life cycle (density, population structure, and reproduction). One of the most interesting findings in this survey concerns the follow-up of the adult Artemia morphology. In fact, we can observe a relationship between physicochemical parameters and all morphological characteristics except the width of the ovisac. Besides, we showed a connection between phytoplankton density and some adult morphological structures.

\section{REFERENCES}

Aмат F. 1980. Differentiation in Artemia strains for Spain. In: The Brine Shrimp Artemia. Morphology, Genetics, Radiobiology, Toxicology (Persoone G., Sorgeloos P., Roels O., Jaspers E., Eds), Vol. 1, pp. 19-39, Universa Press, Wetteren, Belgium.

Амат F. 1982. Diferenciación y distribución de las poblaciones de Artemia de España. III. Oviparismo y ovoviviparismo. Estudio cualitativo y cuantitativo [Differentiation and distribution of Artemia populations in Spain. III. Oviparism and ovoviviparism. Qualitative and quantitative approach]. Invest. Pesq. 46: 3-13 (in Spanish).

Амат F. 1985. Biologia de Artemia [Artemia biology]. Inf. Tec. Inst. Invest. Pesq.: 126-127 (in Spanish).

Amat F., Hontoria F., Navarro T., Gonzalbo E. A., Varo V. I. 1991. Bioecología de Artemia (Crustacea, Branchiopoda) en la Laguna de La Mata, Torre Vieja, Alicante [Bioecology of Artemia (Crustacea, Branchiopoda) in Laguna de La Mata, Torre Vieja, Alicante]. Instituto de Acuicultura, Torre de la Sal (CSIC), España (in Spanish).

Arashrevich E. G., Sapozhnikov P. V., Soloviov K. A., Kudyshinin T. V., Zavialov P. O. 2008. Artemia parthenogenetica (Branchiopoda: Anostraca) from the Large Aral Sea: Abundance, distribution, population structure and cyst production. J. Mar. Syst. doi:10.1016/ j.jmarsys.2008.03.015.

BAID I. 1967. On the development of Artemia salina (L.). J. Bombay Nat. Hist. Soc. 64: 432-439.

Ballardin E., Metalli P. 1963. Osservazioni sulla biologia di Artemia salina (L.). Rend. Ist. Lomb. Sci. Lett. B. 97: 194-254.

Bertalanffy L. V., KrywienczyK J. 1953. The surface rule in crustaceans. Amer. Nat. 87: 107.

Browne R. A. 1982. The costs of reproduction in brine shrimp. Ecology 63: 43-47.

Ben Naceur H., Ben Rejeb Jenhani A., Romdhane M. S. 2009. Ecobiological survey of the brine shrimp Artemia salina from Sabkhet El Adhibet (south-east Tunisia). J. Mar. Biol. Ass. U.K. 89: 1109-1116.

Camargo W. N., Ely J. S., Duran G. C., Sorgeloos P. 2004. Influence of some physicochemichal parameters on Artemia biomass and cyst from the Colombian Caribbean. J. World Aquacult. Soc. 35: 296-305. 
Clegg J. S., Trotman C. N. 2002. Physiological and biochemical aspect of Artemia ecology. In: Artemia: Basic and Applied Biology (Abatzopollos T. J., Beardmore J. A., Clegg J. S., Sorgeloos P., Eds), pp. 129-170, Kluwer Academic Publishers, Netherlands.

D’Agostino A. S., Provasoli L. 1968. Effect of salinity and nutrients on mono- and diaxenic cultures of two strain of Artemia salina. Biol. Bull. 134: 1-14.

Dolapsakis N., Tafas T., Abatzopoulos T. J., Ziller S., Economou-Amilli A. 2005. Abundance and growth response of microalagae at Megalon Embolon solar saltworks in northern Greece. J. Appl. Phycol. 17: 39-49.

DownING J. A. 1984. Assessment of secondary production: the first step. Chapter 1. In: A Manual on Methods for the Assessment of Secondary Productivity in Fresh Waters (DownING J. A., Rigler F. H., Eds), Blackwell Scientific Publications, Oxford, England.

Gaevskaya N. S. 1916. Variability of Artemia salina (L). Russ. Pub. Spec. Zool. Lab. Acad. Sci. 2: $1-37$.

Gajardo G. M., Beardmore J. A. 1989. Ability to switch reproductive mode in Artemia is related to maternal heterozygosity. Mar. Ecol. Prog. Ser. 55: 191-195.

Gajardo G. M., Sorgeloos P., Beardmore J. A. 2006. Inland hypersaline lakes and the brine shrimp Artemia as simple models for biodiversity analysis at the population level. Saline Systems 2: 14 .

Gilchrist B. M. 1960. Growth and form of the brine shrimp Artemia salina (L.). Proc. Zool. Soc. Lond. 134: 221-235.

Gliwicz Z. M., Wurtsbaugh W. A., Ward A. 1995. Brine shrimp ecology in the Great Salt Lake, Utah. June 1994-May 1995. Performance Report to the Utah Division of Wildlife Resources. Salt Lake City, UT.

JeLLISON R. 2005. IX International Conference on Salt Lake research: Research opportunities and management challenges. Saline Systems 1: 2.

Lenz P. H. 1987. Ecological studies on Artemia: a review. In: Artemia research and its applications. Ecology, culturing, use in aquaculture (Sorgeloos P., Bengtson D. A., Decleir W., Jaspers E., Eds), Vol. 3, pp. 5-18. Universa Press, Wetteren, Belgium.

Litvinenko L. I., Boyko E. G. 2008. The morphological characteristics of Artemia shrimps from Siberian populations. Inland Water Biol. 1: 37-45.

Litvinenko L. I., Kozlov A. V., Kovalenko A. I., Bauer D. S. 2007. Salinity of water as a factor to determine the development of the brine shrimp Artemia population in Siberian lakes. Hydrobiologia 576: 95-101.

Marcus N. H. 1984. Variation in the diapause response of Labidocera aestiva (Copepoda: Calanoida) from different latitudes and its importance in evolutionary process. Biol. Bull. 166: $127-139$.

Muñoz J., Gómez A., Green A. J., Figuerola J., Amat F., Rico C. 2008. Phylogeography and local endemism of the native Mediterranean brine shrimp Artemia salina (Branchiopoda: Anostraca). Mol. Ecol. 17: 3160-3177.

NAEgel L. C. A., Rodríguez S. 2002. Ecological observations and biomass proximate composition of the brine shrimp Artemia (Crustacea: Anostraca) from Pichilingue, Baja California Sur, Mexico. Hydrobiologia 486: 185-190.

Román M., Rodríguez A. 1986. Cultivo de Artemia en estanques de salinas de Cádiz (España) [Artemia culture in Salt-ponds of Cádiz (SW of Spain)]. Invest. Pesq. 50: 407-419 (in Spanish).

Romdhane M. S., Ben Chikh N., Ghlala I., Charfi F. 2001. La biodiversité de l'Artemia dans les salines et les sabkha tunisienne [Artemia biodiversity in Tunisian saltwork and sabkha]. International Workshop sur la biodiversité marine. Alger (in French).

Romdhane M. S., Ben Naceur H., Hamrouni S., Ben Rejeb Jenhani A., El Cafsi M. 2004. Biological and biochemical characterisation of Artemia from Tunisian wetlands (Agh N., Sorgeloos P, Eds), International workshop on Artemia. INCO-DEV Project on Artemia biodiversity, pp. 89-91, Urmia, Iran. 
Sorgeloos P., Lavens P., Léger P., Tackaert W., Versichele D. 1986. Manual for the culture and use of brine shrimp Artemia in aquaculture. The Belgian Administration for Development Cooperation. The Food and Agriculture Organization of the United Nations. State University of Ghent, Belgium-Faculty of Agriculture. Belgum.

Sorgeloos P., Dhert P., Candreva P. 2001. Use of the brine shrimp Artemia spp., in marine fish larviculture. Aquaculture 200: 147-151.

Torrentera L., Dodson S. I. 2004. Ecology of the brine shrimp Artemia in the Yucatan, Mexico, Salterns. J. Plankton Res. 26: 617-624.

Triantaphyllidis G. V., Poulopoulou K., Abatzopoulos T. J., Pérez C. A. P., Sorgeloos P. 1995. Salinity effect on survival, maturity, growth, biometrics, reproductive and lifespan characteristics of a bisexual and parthenogenetic population of Artemia (International Study on Artemia XLIX). Hydrobiologia 302: 215-227.

Van Stappen G. 2002. Zoogeography. In: Artemia Basic and Applied Biology (Abatzopoulos T. J. et al. Eds), pp. 171-215, Dordrecht, Kluwer Academic Publishing,

Vanheacke P., Siddall S. E., Sorgeloos P. 1984. Combined effects of temperature and salinity on the survival of Artemia of various geographical origins (International study on Artemia. XXXII). J. Exp. Mar. Biol. Ecol. 80: 259-275.

Van Stappen G., Fayazi G., Sorgeloos P. 2001. Field study of the Artemia urmiana (Günther, 1890) population in Lake Urmiah, Iran (International study on Artemia LXIII). Hydrobiologia 466: $133-143$.

Warren J. K., Kendall C. C. 1985. Comparison of sequences formed in marine sabkha (subaerial) and salina (subaqueous) settings-modern and ancient. Am. Assoc. Pet. Geol. Bull. 69: 10131023 .

Wear R. G., Haslett S. J. 1987. Studies on the biology and ecology of Artemia from Lake Grassmere, New Zeland. In: Artemia Research and Applications. Ecology, Culturing, Use in Aquaculture (Sorgeloos P., Bengtson D. A., Decleir W., Jaspers E., Eds), Vol. 3, pp. 101-126. Universa Press, Wetteren, Belgium. 